SLAC-PUB-8113

April 1999

\title{
High Current, Long Beam Pulse with SLED*
}

\author{
F. J. Decker, Z. D. Farkas, J. Turner \\ Stanford Linear Accelerator Center, Stanford University, Stanford, CA 94309
}

\begin{abstract}
A proposed, high charge, fixed target experiment (E-158) is planned to run with the highest possible energies available at the Stanford Linear Accelerator Center (SLAC), at 45 and $48 \mathrm{Gev}$. The charge is up to $6 \cdot 10^{11}$ particles in a $370 \mathrm{~ns}$ long beam pulse. The SLAC Energy Development (SLED) rf system generates an increasing no-load beam energy, with a linearly decreasing slope. We show how to obtain a current variation that tracks the no-load voltage, resulting in zero energy spread. We discuss the results of a lower energy experiment that verifies the predicted charge and current at the energies required for E-158.
\end{abstract}

Contributed to the IEEE Particle Accelerator Conference, PAC99

New York, NY, 29-Mar-1999 to 2-Apr-1999

${ }^{*}$ Work supported by Department of Energy contract DE-AC03-76SF00515. 


\title{
High Current, Long Beam Pulse with SLED
}

\author{
F. J. Decker, Z. D. Farkas, J. Turner
}

\section{Abstract}

A proposed, high charge, fixed target experiment (E-158) is planned to run with the highest possible energies available at the Stanford Linear Accelerator Center (SLAC), at 45 and $48 \mathrm{Gev}$. The charge is up to $6 \cdot 10^{11}$ particles in a $370 \mathrm{~ns}$ long beam pulse. The SLAC Energy Development (SLED) rf system generates an increasing no-load beam energy, with a linearly decreasing slope. We show how to obtain a current variation that tracks the no-load voltage, resulting in zero energy spread. We discuss the results of a lower energy experiment that verifies the predicted charge and current at the energies required for E-158.

\section{INTRODUCTION}

In steady state the no-load and current induced voltages are constant, hence their difference, the loaded voltage, is also, constant. With SLED, the no-load or rf-generator voltage, $V_{g}(t)$ and the current induced voltage $V_{b}(t)$, vary with time and do not reach steady state.

Let $t_{i}$ be the time when the beam starts, $t_{e}$ when the beam ends, $V_{i}$ the no-load voltage at $t_{i}$, and $V_{g b}$ the noload voltage during the beam pulse minus $V_{i}$. Thus,

$$
V_{i}=V_{g}\left(t_{i}\right), \quad V_{g b}\left(t-t_{i}\right)=V_{g}\left(t-t_{i}\right)-V_{i} .
$$

Reset the starting time to zero by replacing $t-t_{i}$ by $t$. To minimize energy spread, $V_{b}(t)$, should track $V_{g b}(t)$ as closely as posible. To accomplish this we can vary the rf into the accelerator section, or the current or both. Let the no-load voltage at the end of the beam pulse minus the no-load voltage at the beginning of the beam pulse be $V_{d}=V_{g}\left(t_{e}\right)-V_{g}\left(t_{i}\right)$.

Define the optimum current, $I_{o}$, as the current that at the end of a beam pulse of duration $T_{b}$, induces a voltage $V_{b}\left(T_{b}\right)$, equal to $V_{d}$. The loaded voltage as a function of time is $V_{l}(t)=V_{i}+V_{g b}(t)-V_{b}(t)$. Define the loaded voltage $V_{L}$ as the median of $V_{l}(t)$ and the energy spread $d v / v=\left(V_{l}(t)-V_{L}\right) / V_{L}$. With the optimun current there will be, in general, an unacceptable energy spread. We will show that for any variation of $V_{g b}(t)$, we can vary the current so that $V_{b}(t)$ tracks $V_{g b}(t)$ exactly.

The steady state no-load voltage without SLED

$$
V_{g s}=\sqrt{Z_{g} N_{g s} P_{s}}, \quad Z_{g}=\eta_{s} s T_{f} N_{g s} L_{s} .
$$

$N_{g s}$ is the number of sections with $\mathrm{rf}, P_{s}$ is the $\mathrm{rf}$ power into the section, $\eta_{s}$ is the section efficiency, $s(\omega r / Q)$ is the elastance/m, $T_{f}$ is the fill time and $L_{s}$ is the section length. Using the given SLAC parameters, [1, page 117] we obtain for a typical klystron

$$
Z_{g k}=\frac{V_{k}^{2}}{P_{k}}=400 \mathrm{M} \Omega, \quad V_{k}=20 \sqrt{P_{k}}
$$

For the linac $V_{g s}=\sqrt{\eta_{s} s T_{f} N_{g s} P_{s} N_{g s} L_{s}}$.

The shape of $V_{g}(t)$ is determined by the SLED cavities. Let $S_{g}(t)$ be the SLED gain as a function of time, let $S_{g m}$ be its maximum value and let $V_{g m}$ be the maximum SLEDed voltage. Then, the SLEDed voltage as a function of time

$$
V_{g}(t)=S_{g}(t) V_{g s}=\frac{S_{g}(t)}{S_{g m}} V_{g m} .
$$

The SLEDed voltage used in this note is based on an experimentally obtained curve.

\section{OPTIMUM AND ZERO ENERGY SPREAD CURRENTS}

For a step function current, $i_{b}$, passing through an accelerator of length $L_{b}=N_{b s} L_{s}$, the current induced voltage as a function of time $t$ starting at beam injection, is

$$
V_{b}(t)=i_{b} R_{b}(t), \quad R_{b}(t)=\eta_{b}(t) s T_{f} L_{b} / 4 .
$$

For the constant gradient SLAC sections, using $t_{p}=t / T_{f}$,

$$
\begin{aligned}
\eta_{b}(t) & =\frac{1-e^{-2 \tau t_{p}}-t_{p} 2 \tau e^{-2 \tau}}{\tau\left(1-e^{-2 \tau}\right)} & 0 \leq t_{p} \leq 1 \\
& =\eta_{b s}=\frac{1-e^{-2 \tau}-2 \tau e^{-2 \tau}}{\tau\left(1-e^{-2 \tau}\right)} & t_{p} \geq 1
\end{aligned}
$$

The difference of the no-load voltage between the end and the beginning of the beam pulse is

$$
V_{d}=S_{g d} V_{g s}, \quad S_{g d}=S_{g}\left(t_{e}\right)-S_{g}\left(t_{i}\right) .
$$

The beam induced voltage at the end of the beam pulse for a step current

$$
V_{b m}=i_{b} \eta_{b}\left(T_{b}\right) s T_{f} L_{b} / 4 .
$$

Equate $V_{d}$ to $V_{b m}$, solve for $i_{b}$ and obtain

$$
I_{o}=\frac{4 S_{g d}\left(T_{b}\right)}{\eta_{b}\left(T_{b}\right)} \sqrt{\frac{\eta_{s} P_{s}}{s T_{f} L_{s}}} \times \frac{N_{g s}}{N_{b s}} .
$$

In terms of measurable parameters $I_{o}=V_{d} / R_{b}\left(T_{b}\right)$. 
We, now, calculate the current variation that yields a specified self induced beam voltage, in this case, $V_{g b}(t)$. Let $n=1,2,3 \ldots N_{i}$, where $N_{i}$ is the number of injected currents. The interval between current injections is $\Delta t=T_{b} /\left(N_{i}-1\right)$. Let the time when the $n$th current is injected $t_{n}=(n-1) \times \Delta t$. The beam induced voltage at time $t_{n+1}$ is the voltage due to the current injected at time $t_{n}$ plus the voltage due to the $n-1$ previously injected currents. Using $V_{b}\left(t_{n}\right)=V_{g b}\left(t_{n}\right)$ we obtain the injected currents at each point $n$ :

$$
\begin{aligned}
V_{g b}(1) & =0 \\
V_{g b}(2) & =i_{b 1} R_{b}(\Delta t) \\
i_{b 1} & =V_{g b}(2) / R_{b}(\Delta t) \\
V_{g b}(3) & =i_{2} R_{b}(\Delta t)+i_{1} R_{b}(2 \Delta t) \\
i_{2} & =\frac{V_{g b}(3)-i_{1} R_{b}(2 \Delta t)}{R_{b}(\Delta t)} \\
V_{g b}(4) & =i_{3} R_{b}(\Delta t)+i_{1} R_{b}(3 \Delta t)+i_{2} R_{b}(2 \Delta t) \\
i_{3} & =\frac{V_{g b}(4)-\left[i_{1} R_{b}(3 \Delta t)+i_{2} R_{b}(2 \Delta t)\right]}{R_{b}(\Delta t)}
\end{aligned}
$$

For $\quad n>1$

$$
\begin{gathered}
V_{g b}(n+1)=i_{n} R_{b}(\Delta t)+V_{p}(n+1), \\
V_{p}(n+1)=\sum_{p=1}^{n-1} i_{p} R_{b}([n+1-p] \Delta t) . \\
i_{n}=\frac{V_{g b}(n+1)-V_{p}(n+1)}{R_{b}(\Delta t)} . \\
i_{b}(n)=\sum_{j=1}^{n} i_{j} .
\end{gathered}
$$

We can increase the number of injected currents and, consequenly, decrease $\Delta t$ and obtain a continuous total current waveform where, during the beam pulse, $V_{b}(t)$ tracks $V_{g b}(t)$ exactly, resulting in zero energy spread. The zero energy spread average current is about the same as the optimum current. The injected currents are either positive or negative, the the total current is positive as long as $V_{g b}$ does not decrease precipitously.

\section{PREDICTED BEAM CURRENTS AND BEAM ENERGIES}

The no-load voltage, $V_{g}(t)$, is plotted in Fig. 1, as a function of time. We used the previously measured beam energy of the SLEDed linac of $53.7 \mathrm{GeV}$. Also plotted are the current induced and loaded voltages and the energy spread for a 300 ns optimum current pulse starting when the noload voltage is $46 \mathrm{GV}$ and ending $50 \mathrm{~ns}$ before the maximum no-load voltage is reached. The charge is $6.0 \cdot 10^{11}$ particles per pulse about the charge required for E-158. The zero energy spread current for a beam pulse starting the same time as the optimum current pulse but ending $50 \mathrm{~ns}$ after the maximun no-load has been reached and its self induced voltage (dot-dash) are also plotted in Fig. 1. Note that it tracks the no-load voltage. This increases the charge to $6.8 \cdot 10^{11}$ particles per pulse. During the first part of the zero energy spread beam pulse, the current is linearly decreasing, because the slope of the no-load beam energy is linearly decreasing. This, in turn, is because the SLED output that provides the accelerator rf input is, also, nearly linearly decreasing.

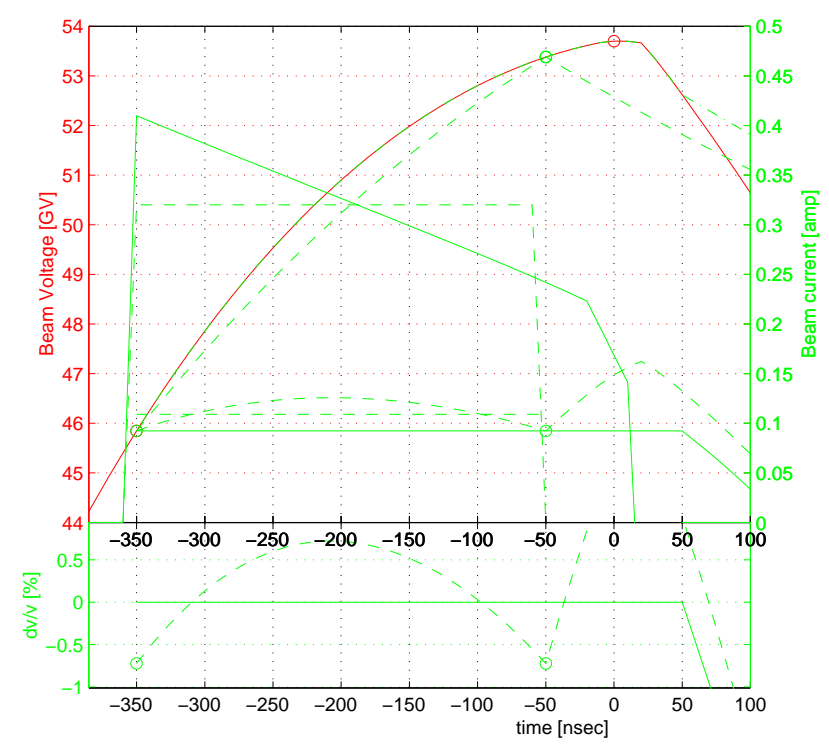

Figure 1: No-load voltage, $V_{g}(t)$, current induced and loaded voltages, $V_{b}(t)$ and $V_{l}(t)$, and the energy spread, $\mathrm{dv} / \mathrm{v}$, for a $300 \mathrm{~ns}$ optimum (dash) and zero energy spread current pulses, left axis; optimum (dash) and zero energy spread currents, right axis. All as a function of time.

The charge per pulse and electrons per pulse are, respectively,

$$
q_{p}=\int_{0}^{T_{b}} i_{b t} d t, \quad e_{p}^{-}=10^{10} \times \frac{q_{p}(\mathrm{nC})}{1.6} .
$$

The beam energy and beam power per pulse and the beam average power are, respectively,

$$
U_{b}=q_{p} V_{i}, \quad P_{b}=U_{b} / T_{b}, \quad P_{b a}=U_{b} \times f_{p r} .
$$

Here, $f_{p r}$ is the pulse repetition frequency. The loaded beam energy and the number of electrons per pulse are plotted in Fig. 2 (top) as a function of beam pulse width. The beam average power at $f_{p r}=120 \mathrm{~Hz}$, and pulse power are also plotted in Fig. 2 (bottom). Their maximum values of 1.6 MW and $22 \mathrm{GW}$, respectively, are reached at a beam pulse width of about $600 \mathrm{~ns}$.

\section{EXPERIMENTAL DATA FOR E-158}

Two test experiments for E-158 were performed. One, with 1 sector $\mathrm{rf}$ and 1 sector beam had a measured energy of 1.2 $\mathrm{GeV}$ and with a sloped beam pulse of $350 \mathrm{~ns}$ had a 

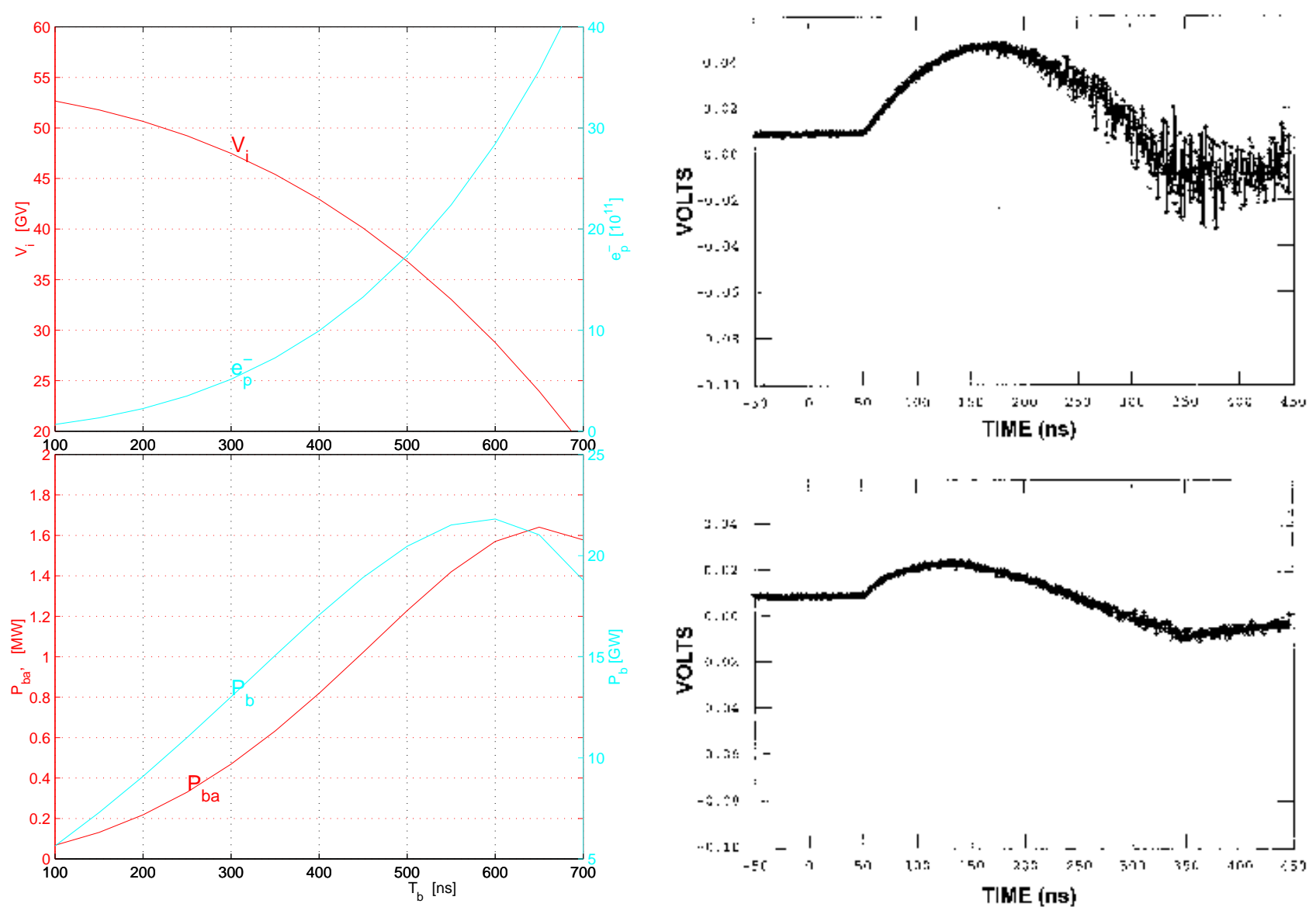

Figure 2: Loaded beam energy and number of electrons per pulse (top), beam average power at 120 pulses per second and beam power per pulse (bottom). All as a function of beam pulse width.

measured charge of $7 \cdot 10^{11}$ particles. Two, with 20 sectors and 30 sector beam the measured energy at the end of 30 sectors was $29 \mathrm{GeV}$ and the charge was $4.5 \cdot 10^{11}$ particles. The ratio of the two charges was 1.56 as predicted by eq. 1 . An energy spread of about $0.25 \%$ was achieved. A small low energy tail could be reduced by a fast phase adjustment of the S-band buncher and capture klystron, but was barely necessary.

\subsection{Transverse Jitter}

The interesting observation was in the transverse plane. The beam position monitors, which integrate over the pulse, should indicate a beam offset by a rising or falling linear slope. A curved slope was observed with an increased jitter at the end of the pulse (see Fig. 3). The curved slope can be explained by a tilted beam pulse and the jitter by the transverse wake fields or dispersion. This tail jitter is correlated with the charge intensity and was up to $2 \mathrm{~mm}$ for a $10 \%$ charge change at the end of the linac. This could be reduced by a factor of five with one corrector making a betatron oscillation to minimize the rms jitter down to about $10 \%$ of the beam size.

Figure 3: The beam position monitor signal (volts, 1 volt $=70 \mu \mathrm{m}$ ) for a $300 \mathrm{~ns}$ beam pulse with a jittery tail, (top) which can be reduced by steering the beam (bottom).

\section{REFERENCES}

[1] R. Neal ed., "The Stanford Two-Mile Accelerator" Benjamin 1968. 